\title{
Determination of Initial Beam Parameters of Varian 2100 CD Linac for Various Therapeutic Electrons Using PRIMO
}

\author{
Reza Maskani ${ }^{1,2 *}$, Mohammad Javad Tahmasebibirgani2,3, Mojtaba Hoseini- \\ Ghahfarokhi $^{1,2}$, Jafar Fatahiasl ${ }^{1,2}$
}

\begin{abstract}
The aim of the present research was to establish primary characteristics of electron beams for a Varian $2100 \mathrm{C} / \mathrm{D}$ linear accelerator with recently developed PRIMO Monte Carlo software and to verify relations between electron energy and dose distribution. To maintain conformity of simulated and measured dose curves within 1\%/1mm, mean energy, Full Width at Half Maximum (FWHM) of energy and focal spot FWHM of initial beam were changed iteratively. Mean and most probable energies were extracted from validated phase spaces and compared with related empirical equation results. To explain the importance of correct estimation of primary energy on a clinical case, computed tomography images of a thorax phantom were imported in PRIMO. Dose distributions and dose volume histogram (DVH) curves were compared between validated and artificial cases with overestimated energy. Initial mean energies were obtained of 6.68,9.73, 13.2 and $16.4 \mathrm{MeV}$ for $6,9,12$ and 15 nominal energies, respectively. Energy FWHM reduced with increase in energy. Three mm focal spot FWHM for $9 \mathrm{MeV}$ and $4 \mathrm{~mm}$ for other energies made proper matches of simulated and measured profiles. In addition, the maximum difference of calculated mean electrons energy at the phantom surface with empirical equation was 2.2 percent. Finally, clear differences in DVH curves of validated and artificial energy were observed as heterogeneity indexes were 0.15 for $7.21 \mathrm{MeV}$ and 0.25 for $6.68 \mathrm{MeV}$. The Monte Carlo model presented in PRIMO for Varian $2100 \mathrm{CD}$ was precisely validated. IAEA polynomial equations estimated mean energy more accurately than a known linear one. Small displacement of R50 changed DVH curves and homogeneity indexes. PRIMO is a user-friendly software which has suitable capabilities to calculate dose distribution in water phantoms or computerized tomographic volumes accurately.
\end{abstract}

Keywords: PRIMO - Monte Carlo simulation - validation - electron beam - energy FWHM

Asian Pac J Cancer Prev, 16 (17), 7795-7801

\section{Introduction}

Electrons are widely used to treat superficial tumors. They deliver an uniform dose in therapeutic ranges and have steep falling that reduce dose to underlying normal tissues (Hogstrom and Almond, 2006). Conventional algorithms in comparison with Monte Carlo (MC) cannot predict precise dose especially in heterogeneous conditions. MC codes, such as EGSnrc/BEAMnrc, MCNP, GEANT and PENELOPE have been developed to calculate dose distributions accurately. (Chetty et al., 2007)

MC Codes need structural details and initial beam parameters as input to predict deposited dose. Structural details include geometry and material of components where beam passes through them. Manufacturers don't report these data clearly because of commercial reasons. Initial beam parameters consist mainly of mean energy, energy spectrum, and spot size of primary electron beam
(Conneely et al., 2013).

Recently, a PENELOPE based MC software called "PRIMO" has been developed which contains preset structural details for some types of Linacs. (Rodriguez et al., 2013) The package offers a user-friendly graphic interface to simulate electron and photon beams in any field sizes. It calculates dose distributions in water phantoms or computerized tomography (CT). PRIMO makes phase-space files in the International Atomic Energy Agency (IAEA) format (Capote et al., 2006) which can be loaded in other MC codes like EGSnrc/BEAMnrc.

Since the early beam parameters are different even in same types of Linac, it's necessary to set them accurately so that simulated and measured data match correctly. Some researchers used simple mono-energetic electron beams to simulate Varian machines by EGS/BEAM. (Huang et al., 2005; Weinberg et al., 2009; Aubry et al., 2011; Connell et al., 2012; Conneely et al., 2013) In addition, based on a report provided by Varian Co (Tanabe and Hamm, 1985). 
Full Width at Half Maximum (FWHM) of energy was assumed constant ( $3 \%$ of mean energy) for all energies in some other works. (Rogers et al., 1995; Keall et al., 2003; Shimozato et al., 2013; Ojala et al., 2014)

Deng et al. estimated energy FHWM 5.3, 4 and 3.9\% for nominal energies 6,12 and $20 \mathrm{MeV}$ respectively (Deng et al., 2001). Furthermore, energy FWHM has been mentioned up to $14 \%$ (Faddegon et al., 2009) and 20\% (Sawkey and Faddegon, 2009) for a Siemens Linac.

Recently, Ojala et al. suggested the FWHM of the focal spot size is less than $1 \mathrm{~mm}$ (Ojala et al., 2014). However, other researchers considered 1 to $2 \mathrm{~mm}$ size for spatial FWHM. (Huang et al., 2005; Connell et al., 2012; Shimozato et al., 2013) Weinberg et al. reported it up to $4.5 \mathrm{~mm}$ in low energies (Weinberg et al., 2009).

To the best of our knowledge, has not been reported any validation study on Varian Linac by using PRIMO software. This study aims to determine initial beam parameters for Varian 2100CD Linac electron beams by PRIMO as well as examine relation between them and depth dose characteristics. Moreover, we examined the effect of measurement error on estimation of primary beam parameters. Afterwards, inaccuracy of treatment planning results was studied in an anthropomorphic phantom.

\section{Materials and Methods}

\section{Experimental measurements}

Electron beams with nominal energies 6,9,12 and 15 $\mathrm{MeV}$ of a Varian 2100CD radiotherapy linear accelerator (Varian Medical Systems, Palo Alto, CA, USA) were used. Measurements were performed using calibrated CC13 cylindrical ion chamber and CU500 electrometer in a computer controlled scanning water phantom (Blue Phantom, Scanditronix Wellhofer). The source-to-surface distance (SSD) was $100 \mathrm{~cm}$ and a $10 \times 10 \mathrm{~cm}^{2}$ Applicator was used in all energies.

Percentage depth ionization (PDI) curves were measured at the central axis of electron beam. They were shifted toward the water surface $(1.8 \mathrm{~mm}$ for 6 and $9 \mathrm{MeV}$; $2 \mathrm{~mm}$ for 12 and $15 \mathrm{MeV}$ ) for correction of effective measuring point (Das et al., 2008).

PDIs were converted to percentage depth dose (PDD) based on water/air mass stopping power ratios (IAEA, 2000) by using Omni Pro-Accept software (Version 6.4; Scanditronix Wellhofer). Thereafter, the main dose features such as surface dose (Ds), ranges of maximum and $50 \%$ of dose (R100\& R50), practical range (Rp) and photon contamination dose (Dx) were extracted from the curves.

For each nominal energy we measured lateral profile at the reference depth $\left(_{\text {dref }}=0.6_{R 50}-0.1 \mathrm{~cm}\right)$ (Equation 1). Curve data were converted to acceptable format to compare with simulated curves.

\section{Simulation setup}

PRIMO (version 0.1.5.1202) was installed on a 24 Cores (Intel Xeon CPU E5-2690) server with Microsoft Windows Operation System. Electron mode of Varian Linac 2100 and $10 \times 10 \mathrm{~cm}^{2}$ applicator was selected for all energies. Material of applicator scrapers was considered zinc alloy and standard insert inside the lowest scarper is made of Cerrotru ( $56 \% \mathrm{Sn}, 44 \% \mathrm{Bi})$. After applicator loading, the jaws were automatically set as specified by the vendor according to beam energy.

As mentioned before, for all nominal energies, the initial electron beam parameters including initial energy, energy FWHM and focal spot FWHM should be set. We started the simulation using default values for initial energy which recommended by developer.

Whole head Linac was simulated at once to produce phase space files in IAEA format just below the applicator (end of S2e segment; $z=97.1 \mathrm{~cm}$ ). Phase space files contain energy, position, direction cosine and charge of each particle.

A homogeneous water phantom was voxelized to a grid of $1 \times 1 \times 0.1 \mathrm{~cm} 3$ along central axis to obtain PDD curves. Lateral dose profile was scored in $0.02 \times 1 \times 1 \mathrm{~cm} 3$ voxels at reference depth.

To reach dose uncertainty below $1 \%$, number of histories (and splitting factor) were set $10^{8}(8)$ and $10^{9}(20)$ for PDD and profile calculation respectively. Transport parameters used in the simulations were: $\mathrm{C} 1=\mathrm{C} 2=0.1$, $\mathrm{WCC}=100 \mathrm{KeV}$ and $\mathrm{WCR}=20 \mathrm{KeV}$. We adopted the cutoff energies for electrons, positrons and photons as following set of values: EABS $(\mathrm{e}-)=\mathrm{EABS}(\mathrm{e}+)=100 \mathrm{KeV}$ and $\operatorname{EABS}(\mathrm{ph})=20 \mathrm{KeV}$.

To evaluate the simulation results, each simulated PDD curve was compared with the relevant measured curve by dose analysis part of the software. In an iterative process, the initial energy was changed and simulation was repeated in so far as both PDD curves coincidence in R50. After optimization of initial energy, the energy FWHM was altered to minimize the discrepancies between simulated and measured PDD curves in most depths. Afterward, we adjusted the focal spot FWHM to achieve closest matching to measured profile.

Finally the Gamma analysis with acceptance criteria of $1 \mathrm{~mm}$ distance-to-agreement (DTA) and $1 \%$ percentage dose difference was conducted to verify simulation results. Essential PDD and profile curve characteristics (Ds, R100, R50, Rp, Dx and field size) were extracted from validated calculation.

\section{Relation between electron energy and dose parameters}

The mean electrons energy at the phantom surface, $\mathrm{E}_{0}$, is the most commonly used parameter for selection of stopping power ratios. (Sorcini et al., 1997)

Two well-known empirical relations between E0 and $\mathrm{R} 50$ are $\mathrm{E}_{0}=2.33 \mathrm{R}_{50}$ (Equation 2) (Khan et al., 1991) and $\mathrm{E}_{0}=0.656+2.059 \mathrm{R}_{50}+0.022 \mathrm{R}_{502}$ (Equation 3) (Andreo et al., 1987; Andreo et al., 1997). E0 and R50 are in MeV and $\mathrm{cm}$, respectively.

Conventional energy-range relationship is (Ep) $=0.22+1.98 \mathrm{Rp}+0.0025 \mathrm{Rp}^{2}$ (Equation 4) $(\mathrm{ICRU}, 1984$; Andreo et al., 1987; Khan et al., 1991)

Where most probable energy, Ep0, and Rp are in $\mathrm{MeV}$ and $\mathrm{cm}$ respectively.

To evaluate relation of PDD characteristics and simulated energy spectrum, phase space should be transferred from below the applicator $(Z=97.1 \mathrm{~cm})$ to phantom surface. Phase space was imported as a source 
in BEAMnrc code (V4 2.4.0) and all particle were passed thought a $2.9 \mathrm{~cm}$ air gap. EGS MC Transport parameters were set to $\mathrm{ECUT}=\mathrm{AE}=521 \mathrm{keV}$ and $\mathrm{PCUT}=\mathrm{AP}=10$ $\mathrm{keV}$. Resulted phase space in IAEA format was restored to PRIMO and was analyzed with $10 \mathrm{keV}$ energy bins. Specifications of energy spectrum including mean energy and most probable energy were derived from a square field wide enough to meet the lateral scatter equilibrium conditions.

\section{Effect of overestimated initial beam energy on treatment planning results}

Correct measurement is essential for proper validation of a MC model. We have observed $\pm 1 \mathrm{~mm}$ differences in determining R50 among various measurements. This difference may be due to change of beam quality over time or misappropriate adjustment of dosimeter. Although these variations are within acceptable range of beam energy constancy (2\%/2 mm) (Klein et al., 2009), they can lead to wrong estimation of initial beam energy.

To show the effect of overestimated initial beam energy on dose distribution, we intentionally shifted measured R50 of $6 \mathrm{MeV}$ electrons as much as $2 \mathrm{~mm}$ downward. Then, corresponding initial beam energy was found by matching calculated and artificial measured R50.

A Thorax Phantom (CIRS Model 002LFC) was used which is made of plastic water, lung and bone sections. The phantom was scanned using 64-slice CT system (SOMATOM Sensation 64 eco; Siemens Healthcare) and DICOM images were imported in the PRIMO program. CT calibration curve (mass density vs. CT number) was adjusted so the final simulated geometry contains only air, lung, soft tissue and compact bone as well as each organ has uniform density.

A hypothetical clinical target volume (CTV) from $7 \mathrm{~mm}$ below the phantom surface to depth of $2 \mathrm{~cm}$ was defined similar to a typical clinical condition. The total volume of the left lung was considered as organs at risk. Dose distribution was calculated for both right and overestimated initial beam energy. Then dose-volume histogram curves were drawn. To compare dose uniformity in both conditions, the homogeneity index (HI) (ICRU 83, 2101) was utilized as $\mathrm{HI}=(\mathrm{D} 2 \%-\mathrm{D} 98 \%) / \mathrm{D} 50 \%$

Where $\mathrm{D} 2 \%$, D98\%, and D50\% are near maximum, near minimum and median dose, respectively.

\section{Results and Discussion}

\section{Tuning of initial beam parameters}

Simulation was started by using default values for initial energy. Although developers have suggested monoenergetic primary beam, simulation results couldn't adapt with measured data. As can be seen in Figure 1 for $6 \mathrm{MeV}$, disagreement reduces by increasing FWHM up to $1.2 \mathrm{MeV}$. Afterward, different focal spot FWHMs was tested for adapting simulated lateral profiles with measurement. Up to $2.8 \%$ differences can be seen for small focal spot FWHM of $15 \mathrm{MeV}$ (Figure 2).

After frequent changes in the initial parameters and performing the simulations, maximum conformity between measured and simulated curves were obtained

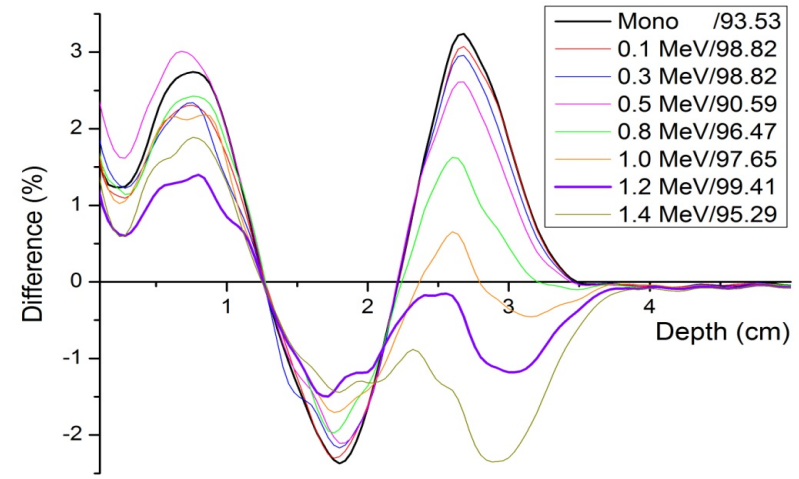

Figure 1. Difference (measured minus simulated) in PDDs from the Energy FWHM Tuning Process for 6 MeV.1.2 MeV was selected as optimized FWHM with $99.41 \%$ points passing the criteria $(1 \% / 1 \mathrm{~mm})$

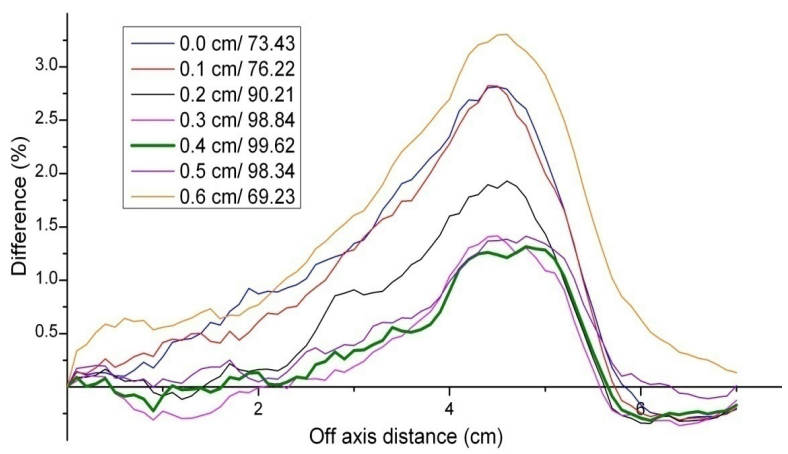

Figure 2. Difference of Lateral Profiles (measured minus simulated) at Reference Depth of $15 \mathrm{MeV}$ for 0-0.6 cm Spatial FWHM. In the inset, numbers at the right of the focal spot FWHM are percentage of points passing the criteria $(1 \% / 1 \mathrm{~mm})$

Table 1. Validated Initial Beam Parameters for Different Nominal Energy

\begin{tabular}{lccc}
\hline $\begin{array}{l}\text { Nominal Energy } \\
(\mathrm{MeV})\end{array}$ & $\begin{array}{c}\text { Initial energy } \\
(\mathrm{MeV})\end{array}$ & $\begin{array}{c}\text { Energy } \\
\text { FWHM(MeV) }\end{array}$ & $\begin{array}{c}\text { Focal spot } \\
\text { FWHM(cm) }\end{array}$ \\
\hline 6 & 6.68 & 1.2 & 0.4 \\
9 & 9.73 & 0.8 & 0.3 \\
12 & 13.2 & 0.6 & 0.4 \\
15 & 16.4 & 0.3 & 0.4 \\
\hline
\end{tabular}
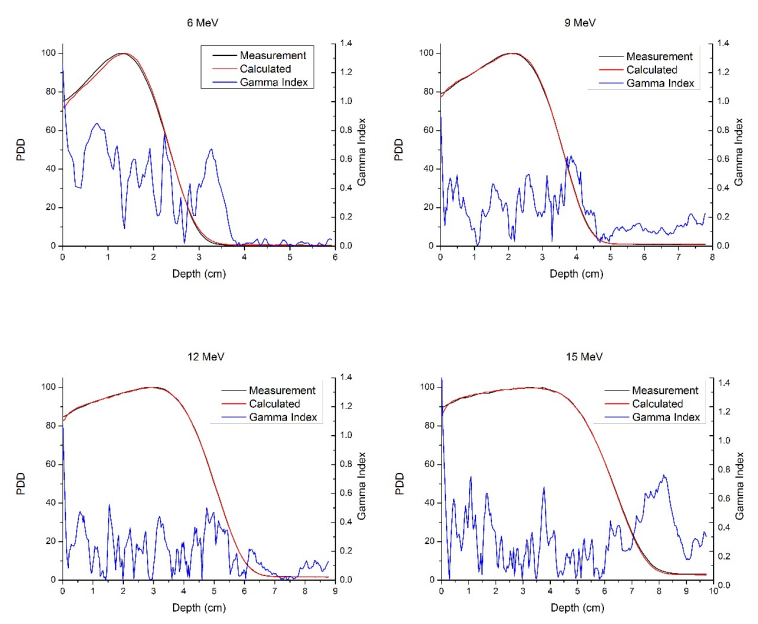

Figure 3. Comparison of Measured and Simulated PDD Curves for Different Nominal Energy 
by using values are listed in Table 1 . Figure 3 indicates PDD curves achieved with these values and related measurements. Gamma indexes versus the depth with $1 \% / 1 \mathrm{~mm}$ criteria have been also shown.

Characteristics of simulated PDD curves compared with the measurement as well as percentage of points passing gamma analysis are presented in Table 2 . The values of Ds and Dx are normalized to the maximum dose. As mentioned earlier, the first step of PDD validation is the R50 matching. It can be seen that difference between the values were less than $0.1 \mathrm{~mm}$. Rp as the next important factor is closely adapted within $0.2 \mathrm{~mm}$. Since the maximum dose of electron PDD, cannot be assigned to just one depth, differences in estimation of R100 values were up to $1.2 \mathrm{~mm}$. A few surface points have gamma index more than one so the most difference was $3.3 \%$ in
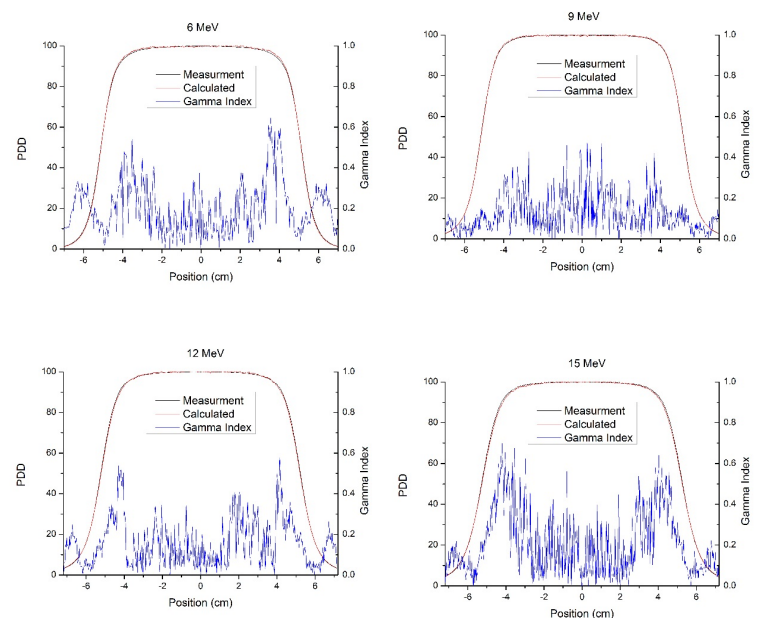

Figure 4. Comparison of Measurement and Simulation Profiles at Reference Depths $(1.27,2.02,2.89$, and 3.7 cm) for6, 9, 12, $15 \mathrm{MeV}$ Respectively. Field size is $10 \times 10$ $\mathrm{cm} 2$ for all cases this region. These differences may be due to the dosimeter volume and originate from measurement error close to air and water border. (Khan et al., 1991; Shimozato et al., 2013)

Average gamma indexes for nominal energies 6 and $9,12,15$ were $0.32,0.23,0.19$ and 0.3 respectively which represents that $12 \mathrm{MeV}$ nominal energy owned the best match. Average gamma indexes before R100 for these energies were $0.7,0.28,0.24$ and 0.31 while for after R100 were $0.22,0.21,0.16$ and 0.29 , respectively. Less average gamma indexes after R100 can be attributed to close match in photon tails. Average gamma indexes before the R100 has risen because maximum gamma indexes have occurred at surface points.

Figure 4 shows measured and simulated lateral profiles at reference depths. Compliance between two profiles has been expressed by gamma analysis results with $1 \% / 1 \mathrm{~mm}$ criteria. Main quantities from profiles and gamma indexes are represented in Table 3. All points of simulated profiles coincided with the measurement by considering above criteria, which validates our simulation for lateral dose

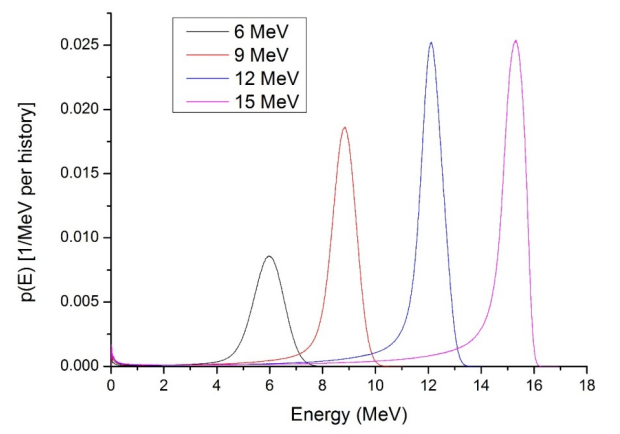

Figure 5. The Energy Spectra of Electrons at the Phantom Surface $($ SSD $=100 \mathrm{~cm})$ and Inside the $7 \times 7$ $\mathrm{cm}^{2}$ Area

Table 2. Measured and Calculated Parameters of PDD Curve. Percentage of Point Shave Passed Gamma Analysis with $1 \% / 1 \mathrm{mmcriteria}$. Ds is dose at the $0.4 \mathrm{~mm}$ depth

\begin{tabular}{|c|c|c|c|c|c|c|c|c|c|c|c|}
\hline Nominal & Ds $(9$ & & R100 & $(\mathrm{cm})$ & R50 & $(\mathrm{cm})$ & $\mathrm{Rp}$ & $(\mathrm{cm})$ & Dx & $(\%)$ & $\begin{array}{c}\text { Passed Percentage } \\
(1 \% / 1 \mathrm{~mm})\end{array}$ \\
\hline Energy (M & V)Meas ${ }^{\mathrm{a}}$ & $\mathrm{Cal}^{\mathrm{b}}$ & Meas & Cal & Meas & $\mathrm{Cal}$ & Meas & $\mathrm{Cal}$ & Meas & $\mathrm{Cal}$ & \\
\hline 6 & 75.54 & 72.24 & 1.32 & 1.36 & 2.35 & 2.36 & 2.97 & 2.96 & 0.54 & 0.5 & 98.66 \\
\hline 9 & 79.38 & 77.71 & 2.11 & 2.05 & 3.6 & 3.59 & 4.4 & 4.38 & 0.72 & 0.94 & 100 \\
\hline 12 & 84.93 & 82.9 & 2.95 & 2.95 & 5 & 5.01 & 6.04 & 6.04 & 1.7 & 1.58 & 99.66 \\
\hline 15 & 89.79 & 86.64 & 3.29 & 3.17 & 6.31 & 6.3 & 7.6 & 7.59 & 3.08 & 2.78 & 99.18 \\
\hline
\end{tabular}

a: Measured, b: Calculated

Table 3. Extracted Field Size and Penumbra from Lateral Profiles. Penumbra was Considered as Distance between The $20 \%$ and $80 \%$ Isodoses. Passed Percentages and Average Gamma Indexes in Different Regions are also Listed

\begin{tabular}{|c|c|c|c|c|c|c|c|c|}
\hline \multirow{2}{*}{$\begin{array}{l}\text { Nominal } \\
\text { Energy } \\
(\mathrm{MeV})\end{array}$} & \multicolumn{2}{|c|}{ Field Width $(\mathrm{cm})$} & \multicolumn{2}{|c|}{ Penumbra $(\mathrm{cm})$} & \multirow{2}{*}{$\begin{array}{c}\text { Average } \\
\text { Gamma } \\
\text { inside } \\
\text { field }\end{array}$} & \multirow{2}{*}{$\begin{array}{c}\text { Average } \\
\text { Gamma } \\
\text { in } \\
\text { penumbra }\end{array}$} & \multirow{2}{*}{$\begin{array}{c}\text { Average } \\
\text { Gamma } \\
\text { outside } \\
\text { field }\end{array}$} & \multirow{2}{*}{$\begin{array}{c}\text { Passed } \\
\text { Percentage } \\
(1 \% / 1 \mathrm{~mm})\end{array}$} \\
\hline & Meas & $\mathrm{Cal}$ & Meas & Cal & & & & \\
\hline 6 & 10.24 & 10.22 & 1.04 & 1.01 & 0.21 & 0.15 & 0.21 & 100 \\
\hline 9 & 10.34 & 10.34 & 1.09 & 1.09 & 0.14 & 0.08 & 0.06 & 100 \\
\hline 12 & 10.46 & 10.42 & 1.22 & 1.25 & 0.18 & 0.13 & 0.14 & 100 \\
\hline 15 & 10.56 & 10.52 & 1.36 & 1.42 & 0.25 & 0.15 & 0.12 & 100 \\
\hline
\end{tabular}

a: Measured; b: Calculated 
Table 4. Mean (E0) and Most Probable Energies (Ep)0 of Simulated Energy Spectra at the Phantom Surface Comparing with Empirical Equations Results

\begin{tabular}{|c|c|c|c|c|c|}
\hline \multirow[t]{2}{*}{ Nominal Energy $(\mathrm{MeV})$} & \multicolumn{3}{|c|}{$\mathrm{E}_{0}(\mathrm{MeV})$} & \multicolumn{2}{|c|}{$\left(\mathrm{E}_{\mathrm{p}}\right)_{0}(\mathrm{MeV})$} \\
\hline & MC Calculated & Equation 2 & Equation 3 & MC Calculated & $\overline{\text { Equation } 4}$ \\
\hline 6 & 5.77 & 5.5 & 5.64 & 5.97 & 6.1 \\
\hline 9 & 8.34 & 8.37 & 8.33 & 8.85 & 8.94 \\
\hline 12 & 11.53 & 11.67 & 11.52 & 12.12 & 12.27 \\
\hline 15 & 14.28 & 14.68 & 14.5 & 15.32 & 15.39 \\
\hline
\end{tabular}

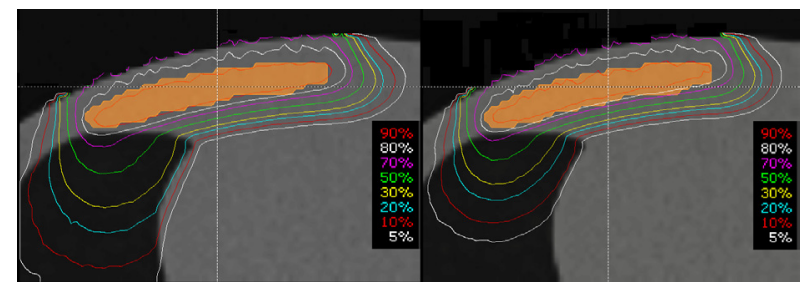

Figure 6. Iso-dose Curves for 6.68 (Right) and 7.21 (Left) MeV for an IMRT Thorax Phantom (CIRS Model 002LFC)

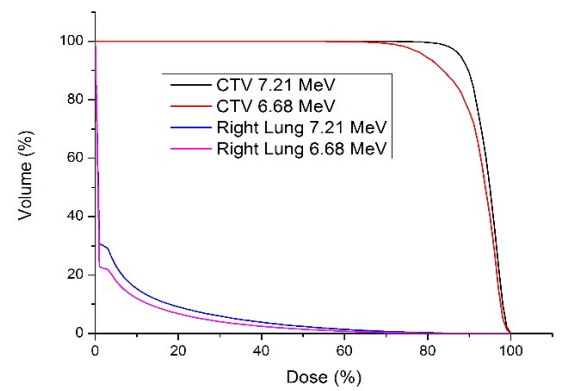

Figure 7. DVHs for CTVs and Right Lung as Organ at Risk in Both Artificial (7.21 MeV) and Real (6.68 MeV) Energy

profiles properly. The average gamma indexes indicate the compatibility of profiles not only in the infield but also in penumbra and outside field regions. The best agreement was achieved in $9 \mathrm{MeV}$ because gamma indexes met the minimum values within all regions. Moreover, all simulated field sizes have differences less than $0.4 \mathrm{~mm}$ with measurement.

According to Table 1 initial electron energies are greater than their nominal values. Nominal energy is an estimate of most probable electrons energy on the surface of the phantom. This difference is due to electrons energy attenuation through their path. In our simulation, the monoenergetic initial beam could not provide a good agreement with measurements. These results are in contrast with some studies which have used simple monoenergetic electron beams. They used simple monoenergetic beams because their works focused on another main aims, for example focal spot determination (Huang et al., 2005; Weinberg et al., 2009), heterogeneity effects (Aubry et al., 2011) and automatic determination of initial parameters (Conneely et al., 2013). It should be noted that their validation criteria haven't clearly been reported.

In some researches energy FWHM has been assumed as a constant percentage (3\%) of initial energy (Rogers et al., 1995; Keall et al., 2003; Shimozato et al., 2013; Ojala et al., 2014). The assumption relays on Tanabe and
Hamm report (Tanabe and Hamm, 1985) who measured energy spectrum at the output of accelerating waveguide of a Varian 1800 Linac. It seems that during electrons pass through the next accelerator parts such as beam transport system and exit window; energy FWHM becomes wider which is notable in the low energy beams. Furthermore, Karzmark et al. stated that typical accepted values of energy FWHM are in the ranges of 3 to 10 percent of initial energy (Karzmark et al., 1993). In current study FWHM of Gaussian energy distribution was achieved 0.3 $\mathrm{MeV}$ for $15 \mathrm{MeV}$ which increased at lower energies (Table 1). Inconsistent with our finding, Deng et al. estimated different energy FHWM as wide as 5.3, 4 and 3.9\% for 6, 12 and $20 \mathrm{MeV}$ nominal energies, respectively (Deng et al., 2001). However, Wider energy FWHM has been mentioned for Siemens Linac (Faddegon et al., 2009; Sawkey and Faddegon, 2009).

The results (Table 1) show that simulation using 3 $\mathrm{mm}$ focal spot FWHM for $9 \mathrm{MeV}$ and $4 \mathrm{~mm}$ for other energy guarantee appropriate match with measurements. This result is in accepted range ( 2 to $4 \mathrm{~mm}$ ) expressed by Karzmark et al. (Karzmark et al., 1993) Our findings are larger than $0.7 \mathrm{~mm}$ (Ojala et al., 2014) and 1.5 to $2 \mathrm{~mm}$ (Shimozato et al., 2013) FWHM which has been reported recently. On the other hand, Weinberg et al. stated that optimal FWHM are $4.5 \mathrm{~mm}$ for 6 and $9 \mathrm{MeV}, 2.2$ to 2.5 $\mathrm{mm}$ for higher energies (Weinberg et al., 2009). The inconsistencies of various simulated models in determined spatial FWHM may be due to differences between geometric details like precise thickness of scattering foils.

\section{Characterization of energy spectrum}

Figure 5 shows the electrons energy spectrum extracted from the phase space files at the phantom surface. In the lower energies because of more scattering and beam divergence, less particles pass through intended area, so shorter peaks has been appeared.

Electrons energy reduces passing through different parts of the Linac head including scattering foils and ion chamber, therefore the most probable energy (spectrum peak) is less than initial energy listed in Table 1. In addition, compared to the initial energy spectrum, FWHMs have increased and scattered low energy electrons from the beam defining components are added. At higher energies which had narrower FWHM, these low-energy electrons created a clear tail and ruined Gaussian symmetry more around most probable energy. The tail increase difference between the mean $\left(\mathrm{E}_{0}\right)$ and most probable energies (Ep) that can be seen in Table 4.

MC Calculated $\mathrm{E}_{0}$ values are closer to results calculated by Equation 3 than Equation 2. The maximum 
difference by Equation 3 was $2.2 \%$ in $6 \mathrm{MeV}$. Ding et al. in a similar study calculated $\mathrm{E}_{0}$ with $4 \%$ differences of those resulted from Equation 2 and 3 for a Linac 2100C. (Ding et al., 1996)

As mentioned by Andreo et al. since Equation 2 has been obtained with a constant source to chamber distance (Andreo et al., 1997), it is not strictly valid for constant SSD measurements. However, TG 70 (Gerbi et al., 2009) as a supplementary of TG 25 has recommended Equation 3 for determination of $\mathrm{E}_{0}$.

$\mathrm{MC}$ calculated (Ep) for all energies is slightly less than Equation 4 results. This difference was also observed by Ding et al. (about 2\%) (Ding et al., 1996). Björk et al. examined initial beams with various spectral width and found that best agreement between MC and Equation 4 achieved for the monoenergetic beam (Björk et al., 2002). It seems the discrepancies are because of the fact that Equation 4 originally are based on measurements from an almost monoenergetic microtron accelerator.

\section{Misestimated initial energy affects treatment planning results}

$\mathrm{R}_{50}$ of validated $6 \mathrm{MeV}$ electrons $(2.35 \mathrm{~cm})$ was shifted $0.2 \mathrm{~cm}$ downward. After iterative simulations, the corresponding initial energy was found as $7.21 \mathrm{MeV}$. Iso-dose curves for 6.68 (real) and 7.21 (artificial) $\mathrm{MeV}$ initial beam energy are illustrated in Figure 6. For 7.21 $\mathrm{MeV}$ iso-dose curves were shifted to under depths. It is quite obvious in lung tissue.

Dose-Volume Histograms (DVHs) has been displayed for both conditions (figure 7). At a glance it seems that CTVs have a similar coverage within $90 \%$ iso-dose in Figure 6, but there are clear differences in DVH curves. With 7.21 and $6.68 \mathrm{MeV}$ energy, $89.22 \%$ and $75.97 \%$ of tumor volume have received at least $90 \%$ of maximum dose, respectively. Planning by using 7.21 MeV (artificial energy) yields a near-ideal DVH curve, while during treatment, patient receives dose whose DVH is attributed to $6.68 \mathrm{MeV}$. As defined before, heterogeneity index (HI) was calculated 0.15 for $7.21 \mathrm{MeV}$ and 0.25 for $6.68 \mathrm{MeV}$. Indeed, the uniformity of dose which will be delivered to CTV is less than that has been planned. Moreover, DVH of organ at risk (right lung) has altered because of iso-dose curves shift (Figure 7).

\section{Conclusion}

Appropriate values of initial parameters for electron beam with 6,9,12 and $15 \mathrm{MeV}$ nominal energies from a Varian 2100 CD Linac were determined using PRIMO, a recently developed MC package. Initial beam energy and energy FWHM were found by matching simulated and measured depth dose cures considering $1 \% / 1 \mathrm{~mm}$ acceptance criteria. Subsequently, focal spot FWHM was defined so that simulated dose profiles were fitted on related measurement. The results suggest monoenergetic beam didn't comply with passing criteria as well as spatial FWHM were nearly equal for all energies. Electrons mean and most probable energy at the phantom surface was extracted from validated phase space file. Polynomial equation of IAEA is more accurate than linear one (TG) in prediction of mean energy. The effect of overestimated initial energy on dose distribution was evaluated in a heterogeneous CIRS phantom. Only $2 \mathrm{~mm}$ displacement of $\mathrm{R}_{50}$ caused change in DVH curves and homogeneity indexes. Therefore, R50 should be carefully measured to avoid errors in determination of initial energy. PRIMO is a user-friendly MC software that can simulate Linac head and calculate dose distribution in either homogeneous or CT based phantoms accurately by proper choosing of the initial beam parameters.

\section{Acknowledgements}

This work was financially supported by grant: (4797) from Vice Chancellor for Research Affairs of Ahvaz Jundishapur University of Medical Sciences.

\section{References}

ICRU (2010). Prescribing, Recording, and Reporting PhotonBeam Intensity-Modulated Radiation Therapy (IMRT). Journal of the ICRU, 10, NP.

IAEA (2000). Absorbed dose determination in external beam radiotherapy: an international code of practice for dosimetry based on standards of absorbed dose to water, Internat. Atomic Energy Agency.

Aubry J-F, Bouchard H, Bessières I, et al (2011). Validation of an electron Monte Carlo dose calculation algorithm in the presence of heterogeneities using EGSnrc and radiochromic film measurements. Journal of Applied Clinical Medical Physics, 12.

Björk P, Knöös T, Nilsson P(2002). Influence of initial electron beam characteristics on Monte Carlo calculated absorbed dose distributions for linear accelerator electron beams. Physics in medicine and biology, 47, 4019.

Capote R, Jeraj R, Ma C, et al (2006). Phase-space database for external beam radiotherapy. IAEA, Nucl. Data Sec. Report INDC (NDS)-0484, International Atomic Energy Agency, Vienna, Austria.

Chetty IJ, Curran B, Cygler JE, et al (2007). Report of the AAPM Task Group No. 105: Issues associated with clinical implementation of Monte Carlo-based photon and electron external beam treatment planning. Medical Physics, 34, 4818.

Conneely E, Alexander A, Stroian G, et al (2013). An investigation into the use of MMCTP to tune accelerator source parameters and testing its clinical application. J App Clinical Medical Physics, 14.

Connell T, Alexander A, Evans M, et al (2012). An experimental feasibility study on the use of scattering foil free beams for modulated electron radiotherapy. Phys Med Biol, 57, 3259-72.

Das IJ, Cheng C-W, Watts RJ, et al (2008). Accelerator beam data commissioning equipment and procedures: report of the TG-106 of the Therapy Physics Committee of the AAPM. Medical physics, 35, 4186-215.

Deng J, Jiang SB, Pawlicki T, et al (2001). Derivation of electron and photon energy spectra from electron beam central axis depth dose curves. Phys Med Biol, 46, 1429-49.

Ding G, Rogers D, Mackie T (1996). Mean energy, energy range relationships and depth scaling factors for clinical electron beams. Medical physics, 23, 361-76.

Faddegon BA, Sawkey D, O'Shea T, et al (2009). Treatment head disassembly to improve the accuracy of large electron field simulation. Medical physics, 36, 4577. 
Gerbi BJ, Antolak JA, Deibel FC, et al (2009). Recommendations for clinical electron beam dosimetry: Supplement to the recommendations of Task Group 25. Medical physics, 36, 3239-79.

Hogstrom KR, Almond PR (2006). Review of electron beam therapy physics. Phys Med Biol, 51, 455-89.

Huang VW, Seuntjens J, Devic S, et al (2005). Experimental determination of electron source parameters for accurate Monte Carlo calculation of large field electron therapy. Physics in medicine and biology, 50, 779.

ICRU RD (1984). Electron beams with energies between 1 and $50 \mathrm{MeV}$. ICRU Report, 35.

Karzmark C, Nunan CS, Tanabe E 1993. Medical electron accelerators, McGraw-Hill.

Keall PJ, Siebers JV, Libby B, et al (2003). Determining the incident electron fluence for Monte Carlo-based photon treatment planning using a standard measured data set. Medical physics, 30, 574.

Khan FM, Doppke KP, Hogstrom KR, et al (1991). Clinical electron beam dosimetry: Report of AAPM Radiation Therapy Committee Task Group No. 25 tg. Medical Physics, 18, 73-109.

Klein EE, Hanley J, Bayouth J, et al (2009). Task Group 142 report: Quality assurance of medical acceleratorsa). Medical physics, 36, 4197-212.

Ojala J, Hyödynmaa S, Barańczyk R, et al (2014). Performance of two commercial electron beam algorithms over regions close to the lung-mediastinum interface, against Monte Carlo simulation and point dosimetry in virtual and anthropomorphic phantoms. Physica Medica, 30, 147-54.

Rodriguez M, Sempau J, Brualla L (2013). PRIMO: A graphical environment for the Monte Carlo simulation of Varian and Elekta linacs. Strahlentherapie Onkologie, 189, 881-6.

Rogers D, Faddegon B, Ding G, et al (1995). BEAM: A Monte Carlo code to simulate radiotherapy treatment units. Medical physics, 22, 503.

Sawkey D, Faddegon B (2009). Determination of electron energy, spectral width, and beam divergence at the exit window for clinical megavoltage x-ray beams. Medical Physics, 36, 698.

Shimozato T, Okudaira K, Fuse H, et al (2013). Monte Carlo simulation and measurement of radiation leakage from applicators used in external electron radiotherapy. Physica Medica, 29, 388-96.

Sorcini BB, Hyodynmaa S, Brahme A (1997). Quantification of mean energy and photon contamination for accurate dosimetry of high-energy electron beams. Phys Med Biol, 42, 1849-73.

Tanabe E, Hamm RW (1985). Compact multi-energy electron linear accelerators. Nuclear Instruments and Methods in Physics Research Section B: Beam Interactions with Materials and Atoms, 10, 871-6.

Weinberg R, Antolak JA, Starkschall G, et al (2009). Influence of source parameters on large-field electron beam profiles calculated using Monte Carlo methods. Phys Med Biol, $\mathbf{5 4}, 105$. 